\title{
Quantum-Assisted Retinal Drusen Detection Algorithm using Entropy-Based Image Processing Techniques
}

\author{
L. Jeganson Durai, J. Vijayakumar
}

\begin{abstract}
Drusen identification is the fundamental operation in the automated diagnosis of eye diseases. Manual and automatic detection of the drusen in the retinal fundus images has been developed recently in the classical manner only. This work provides the quantum-based retinal drusen detection method using entropy-based image processing techniques. This algorithm is the composite system of two channels, classical and quantum channels for the preprocessing and drusen detection respectively. This research work has been evaluated with the databases of DRIVE, STARE, MESSIDOR, E-Optha-EX and ONH-Hunter. This quantum-based approach will be analyzed with the results of the existing classical methods and proves its efficiency from the calculations of sensitivity, specificity, accuracy and execution time.
\end{abstract}

Keywords: Drusen, Quantum Information Processing, Entropy-based image processing

\section{INTRODUCTION}

$\mathrm{D}$ rusen is one of the retinal anatomies and the main intimation of eye diseases. They are the yellow deposits which can be observed manually from the retinal fundus image, which is shown in Fig. 1, the marked regions are the drusen presented area.

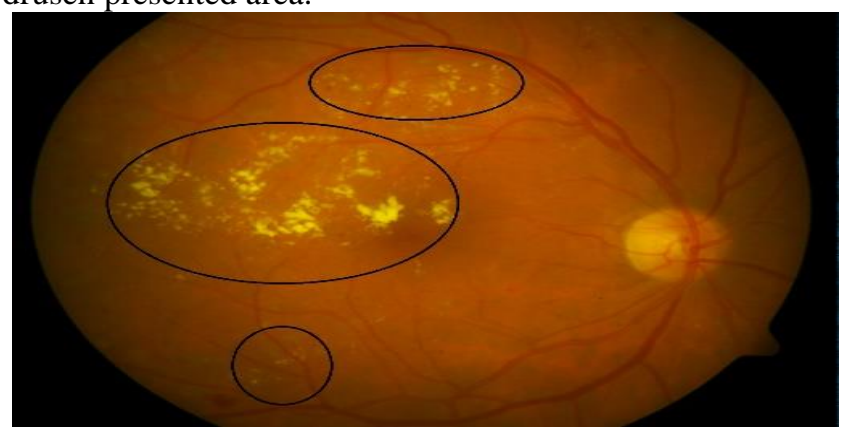

Fig. 1.Drusen presence (Yellow deposits) in Retinal Fundus Image.

Revised Manuscript Received on February 05, 2020.

* Correspondence Author

L. Jeganson Durai*, Department of Electronics and Instrumentation, Bharathiar University, Coimbatore, India.

E-mail: jegansondurai@gmail.com

J. Vijayakumar, Department of Electronics and Instrumentation, Bharathiar University, Coimbatore, India. E-mail: vijayakumar@buc.edu.in

(C) The Authors. Published by Blue Eyes Intelligence Engineering and Sciences Publication (BEIESP). This is an open access article under the CC BY-NC-ND license (http://creativecommons.org/licenses/by-nc-nd/4.0/)
But the automated computational techniques are needed to the doctors for the fast and more accurate analysis of the drusen measurement. Many approaches are developed by the researchers, which are reviewed in the next section. They are mostly based on classical properties, but this research work provides the Quantum-based image processing approach, which is robust, rapid and more efficient than the classical systems.

First, the preprocessing steps have been developed classically. The green channel extraction, denoising operation using Butterworth filter and adaptive histogram equalization methods are used to the noiseless and more contrasted image for the input what will be given. The blood vessels are presented in the foreground of the retinal image, so it is also considered as the noise, they will be eliminated after its segmentation. Secondly, the image will be processed in the quantum channel, which means the Bit values of the pixels will be transformed into Qubit values. In the quantum manner, the entropy-based method is suggested for the feature extraction and an automated double thresholding method will be used to the identification of the retinal drusen and other subretinal deposits. Finally, the performance of the proposed algorithm will be analyzed with the existing classical methods and evaluated. The execution time will be measured and prove its fast and highly coherent properties than the classical approaches.

\section{LITERATURE REVIEW}

The quantum-based identification of drusen is the main aim of this research problem. The drusen detection methods and quantum image processing related approaches are analyzed as follows: CNN-based drusen detection algorithm is presented [1], which have the functions of noise removal, histogram equalization, segmentation and provides the more appropriate detection of the subretinal deposits. An automatic drusen identification method is proposed [2] to the diagnosis of AMD. Green channel and ROI extraction and blood vessel removal are followed in the preprocessing steps and Renyi's entropy thresholding algorithm is used to the drusen detection. Another automatic drusen detection and segmentation method are suggested [3] using the gradient-based segmentation, connected component labelling and edge lining. The potential drusen areas are detected using the composite model [4], which consists of the local intensity distribution, adaptive thresholding and edge detection. 
An automatic drusen segmentation algorithm [5] is designed for the SD-OCT images.

The quality of the detected drusen will be enhanced using the retinal projection method. Different patterns of the drusen are obtained using the Summed-voxel projection (SVP). A fuzzy logic-based drusen detection method is approached [6] in the retinal angiographic images. The detected drusen are quantified using the image optimal partition, fuzzification and segmentation. Quantum measurement-based edge detection and denoising algorithms are suggested [7], which follows the QSP framework. The results show the clean edge map to the image and the proposed quantum-based filters perform the denoising function precisely than existing methods.

A quantum version of the image segmentation algorithm is developed [8] using thresholding-based operations. This work analyzed the applications of quantum image processing and prove their efficiency than classical approaches. An optimal scheme is proposed [9] using the real-coded quantum genetic algorithm. Curvelet transform is used, which gives better performance than the wavelet-transform and its coefficients are reformed using the non-linear enhancement mapping functions. An object extraction method is presented [10] with the numerical form of the quantum mechanics equations. For the most part is the Schrodinger equation, which has a very powerful mathematical structure and is enough to the analysis of the complex objects and its features. A quantum mechanics-based contour extraction method is proposed and evaluated [11] by the medical images. It performs fast and reduces the computational cost. Mainly it will be used for its robustness to the low contrast images. An algorithm for the multiobjective image segmentation is suggested [12] using the quantum information theory, which performs under the basis of K-means classical algorithm.

\section{METHODOLOGY}

Fig.2 demonstrates the total process of the proposed Drusen extraction and localization algorithm. The retinal fundus images will be given as the input image, they are collected from various online available datasets.

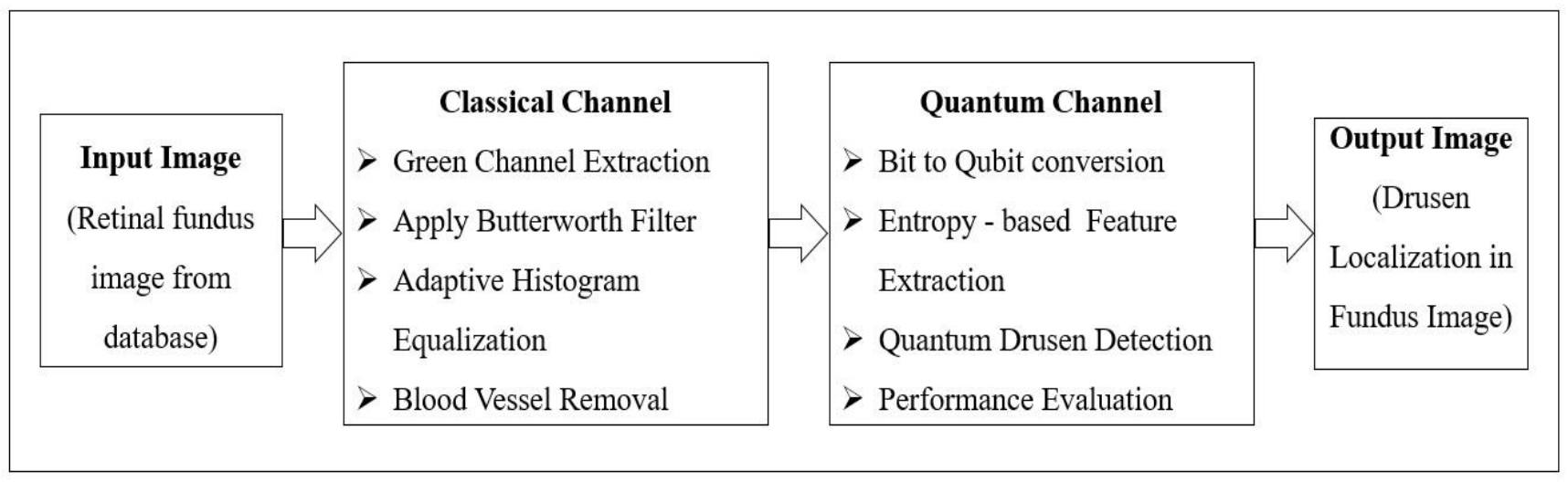

Fig. 2.Block diagram for the Quantum-Assisted Retinal Drusen detection algorithm.

Green channel extraction process will be suggested to get more accurate results and visible features, because of the number of green colour pixels are greater than red and blue pixels. Images are noisy in natural, so the unwanted noises should be reduced using digital filters. High-pass Butterworth filter is preferred for this research problem to get more accuracy and rapid performance. Now, the image will be noiseless one, but the sharpness of the features may be affected. So, Adaptive Histogram Equalization technique is used to increase the contrast of the retinal image.

Now, the image is pre-processed and it will be the noiseless and contrast-enhanced image. Retinal blood vessels are placed in the foreground in the fundus image, which should be neglected for the clear-cut detection of the drusen area. The blood vessels will be identified and eliminated from the image. The moral of this algorithm is to create the quantum assisted drusen detection method from the retinal image. The retinal image will be transformed from the classical manner into quantum manner. The bits are converted into qubit pixel values. Entropy is the random variable measurement, which is used to characterize the retinal image. High and low levels of entropy range values will be given, the numerical equations will support the automated threshold value selection. In this stage, the drusen will be extracted but we should localize the drusen in the area of an input image. So, we have to find the boundary of the extracted drusen, Canny edge detector is used to find the edges and an outer layer of the optic disc. Finally, the drusen will be localized in the retinal fundus image. The performance of this suggested algorithm should be analysed using the numerical equations of physical parameters such as accuracy, sensitivity, specificity and execution time.

\section{A. Classical Channel}

Step 1: The input of the developed quantum-assisted algorithms is the retinal fundus image, which is represented as $\mathrm{I}\left(\mathrm{m}_{v} \mathrm{n}\right)$, where $\mathrm{m}$ and $\mathrm{n}$ are the numbers of rows and columns respectively. The size of the retinal images obtained from the various databases may be the same or not. For our convenience, we should resize the image into $N X N$ (our standard size $256 \times 256$ ), they are rows and columns of the image, which are specified by any vectors.

Step 2: The green channel extracted image $I_{G}(m, n)$ is defined as,

$$
I_{G}(m, n)=I(m, n)(:,: ; 2)
$$

Step 3: The retinal fundus images are noisy in natural. So, we should filter the image to get more precious and error-free results.

Published By: 
In this research work, the high-pass Butterworth filter is used to reduce the noises of the image. $I_{\omega}$ is cutoff frequency, then the transfer function of the nth order Butterworth filter is

$I_{B W}(m, n)=\frac{1}{1+\left[I_{\omega} / I_{G}(m, n)\right] 2^{n}}$

Step 4: From the filtering process, the noises of the images are not only reduced but also the sharpness of the features is decreased. So, the Adaptive Histogram Equalization technique is used to increase the contrast of the retinal images, which is more precious than other enhancement techniques. The $H(m, n)$ is the equalization factor, which is convoluted with the filtered image, then we get the contrast-enhanced image $I_{H}(m, n)$ and it is described as

$$
I_{H}(m, n)=I_{B W}(m, n) \otimes H(m, n)
$$

The range of the pixel intensity values will be changed by the contrast stretching process. This normalization process $I_{H N}(m, n)$ on the contrast-enhanced image is defined as follows,

$I_{H N}(m, n)=I_{H}(m, n) / m * n$

Here the image, $I_{H N}(m, n)$ will be the noiseless and contrast-enhanced image. Now, we have to proceed with this image as the input image of our proposed algorithm.

Step 5: For the blood vessel removal operation, first they have to be identified. Consider the threshold value as $\mathrm{t}$, which will be taken between 0 and 255. The equations for the entropy of the white and black pixels are (5) and (6) as follows:

$$
\begin{aligned}
H_{w}(t) & =-\sum_{i=0}^{t} I_{m, n}(i) \log \left(I_{m, n}(i)\right) \\
H_{b}(t) & =-\sum_{i=t+1}^{255} I_{m, n}(i) \log \left(I_{m, n}(i)\right)
\end{aligned}
$$

The threshold can be selected by increasing the entropy of black and white pixels:

$$
T=\underset{t=0,1, \ldots 255}{\operatorname{Arg}}\left\{\operatorname{Max}\left(H_{w}(t)+H_{b}(t)\right)\right\}
$$

The thresholding operator $\mathrm{T}$, will be convoluted with the filtered image $I_{f}(m, n)$ as follows,

$I_{t}(m, n)=I_{f}(m, n) \otimes T$

Step 6: Here $I_{t}(m, n)$ is the segmented retinal blood vessels from the fundus image. Now, the retinal blood vessels should be eliminated using under the equation for the accurate drusen localization.

$I(m, n)=I_{H N}(m, n)-I_{t}(m, n)$

\section{B. Quantum Channel}

Step 7: The quantum-assisted algorithm is proposed for the detection of drusen from the retinal fundus image, which is the main difference from other drusen detection techniques.
Normally, the images are classically derived by the bits. For the quantum channel, the bits will be transformed into Qubits (Quantum Bits).

$I(m, n)$ will be converted into $|Q(m, n)\rangle$ assigned to the range of $[0,1]$, which is the superposition of two quantum states $I(m, n)$. These quantum states are described in the orthogonal vector space, $|0\rangle=\frac{1}{0}$ and $|1\rangle={ }_{1}^{0}$. In other words, $|0\rangle$ and $|1\rangle$ are considered to be the drusen and non-drusen pixel representations in this problem. This qubit state has the two basis vectors, so the representation of the pixel in the quantum channel will give more information and accuracy than classical manner. The quantum image $|Q(m, n)\rangle$ is defined as,

$Q|(m, n)\rangle=C_{0}|0\rangle+C_{1}|1\rangle$

Here $\left|C_{0}\right|^{2}+\left|C_{1}\right|^{2}=1$ and the $C_{0}$ and $C_{1}$ are the probability amplitude of the $|0\rangle$ and $|1\rangle$, they will be calculated by the following equations

$\left|C_{0}\right|=\frac{1}{1+e \sqrt{1-\operatorname{mag}(|Q(m, n)\rangle)}}$

and

$\left|C_{1}\right|=\frac{1}{1+e \sqrt{1-\operatorname{mag}(|Q(m, n)\rangle)}}$

In the above equation, $\operatorname{mag}(|Q(m, n)\rangle)$ is the gradient vector magnitude.

Step 8: An Entropy-based thresholding technique is used to localize the drusen from the retinal image. Entropy is the random variable measurement and the entropy of the image $|Q(m, n)\rangle$ is

$$
\text { Entropy }=-\sum|Q(m, n)\rangle \cdot \log _{2}|Q(m, n)\rangle
$$

As per the pixel values of the retinal image many random variables are there, which are described by the Cumulative Distributive Function (CDF). Let assume $\mathrm{X}$ is the random variable and is denoted as the CDF of $\mathrm{X}$. For an automated selection of entropy thresholding, we will calculate the lower-level and higher-level entropy ranges. Those two values will be compared, then we can finalize the threshold value $(\mathrm{T})$.

The transfer function of the low and high range entropy equations are as follows,

$$
\begin{aligned}
& \left.\left.\left.E_{\text {Low }}=-\sum_{u=0}^{M} \sum_{V=0}^{N} Q(m, n)\right\rangle / F_{X}|(m, n)\rangle \cdot \log _{2} Q(m, n)\right\rangle / F_{X}(m, n)\right\rangle(13) \\
& \left.\left.\left.E_{\text {High }}=-\sum_{u=0}^{M} \sum_{V=0}^{N} Q(m, n)\right\rangle / 1-F_{X}|(m, n)\rangle \cdot \log _{2} Q(m, n)\right\rangle / 1-F_{X}(m, n)\right\rangle
\end{aligned}
$$

The low and high range entropies are calculated from the above equations (13) and (14), 
now we have to find the best threshold value $\mathrm{T}$ using the comparisons of $E_{\text {Low }}$ and $E_{\text {High }}$ values, $T=E_{\text {Low }} \approx E_{\text {High }}$. The entropy-based thresholded image $Q_{T}(m, n)$ is clarified by the below conditions from (15), eventually, the drusen are segmented from the retinal fundus image.

$Q_{T}(|m, n\rangle)<T=0$

$Q_{T}(|m, n\rangle)>T=1$

Step 9: After the drusen localization, the boundaries of the detected features will be identified using edge detection. In this research work, drusen localized image is processed with Canny edge detector in horizontal and vertical directions to get 1st order derivative operator $G_{U}$ and $G_{V}$ respectively. The edge gradient value $G_{e}$ is $\sqrt{G_{u}^{2}+G_{v}^{2}}$.

$Q_{E}|(m, n)\rangle=Q_{T}|(m, n)\rangle \otimes G_{e}-\eta$

In this equation, $Q_{E}|(m, n)\rangle$ is the edge detected image and $\eta$ is non-maximum suppression factor, which should be reduced to the unwanted detected edges. Finally, the extracted features of the drusen will be marked in the input fundus image.

Step 10: The sensitivity, specificity, accuracy and percentage of error values should be calculated for measuring the efficiency of the system and those equations are as follows

$$
\begin{aligned}
& \text { Sensitivity }=\frac{T P}{T P+F N} \\
& \text { Specificity }=\frac{T P}{T P+F N} \\
& \text { Accuracy }=\frac{T P}{T P+F N}
\end{aligned}
$$

\section{RESULTS AND DISCUSSIONS}

The numerical solutions of the proposed quantum-assisted drusen detection algorithm from the methodology will be simulated using MATLAB R2013b software platform. The possible outcomes for classical and quantum channels are discussed in this section. Fig. 3 shows the results of pre-processing steps, which includes RGB channel extraction, Filtering, contrast enhancement. First, red, green and blue channels are extracted from the input retinal image, while this process green channel pixel elements are visually comprehensible than red and blue channels. The denoising process is effectuated using Gaussian, Median and Butterworth filters. From this analysis, the Butterworth filter achieves more accurate than others. Then, the contrast of the fundus image will be enhanced with adaptive histogram equalization. The histogram results for input fundus image and pre-processed image are shown in Fig. 3, which reveals the pre-processed image noiseless and contrast-enhanced one.
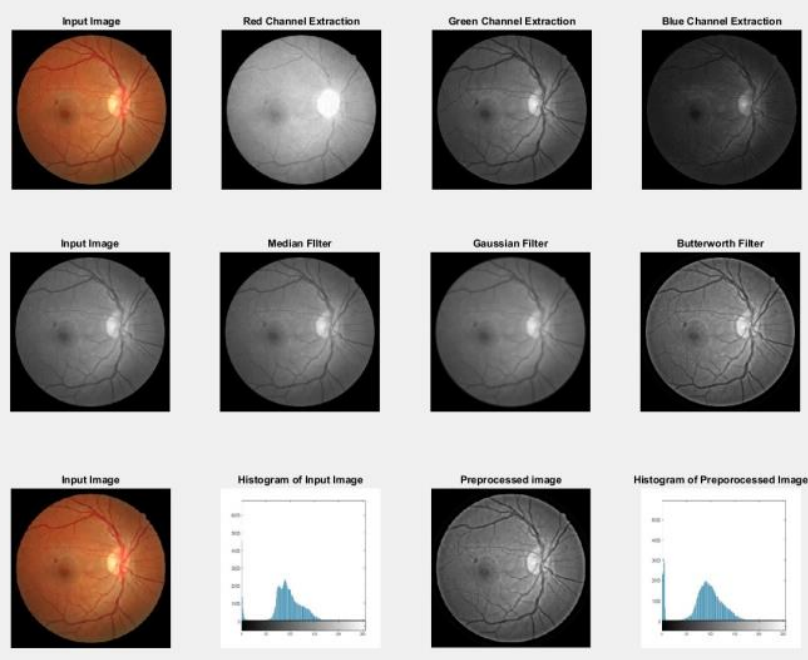

Fig. 3.The results for Pre-processing steps (RGB channel Extraction, Filters (Median, Gaussian, Butterworth), Histogram Equalization)
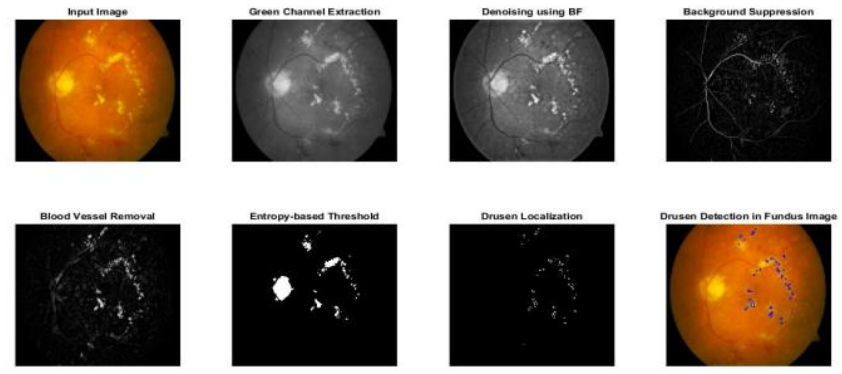

Fig. 4.The results for quantum-assisted retinal drusen detection algorithm using entropy-based image processing techniques

The results for quantum-assisted retinal drusen detection algorithm using entropy-based image processing techniques in Fig. 4. The green channel of retinal fundus image extracted (1) for a greater number of pixels occurrence. Butterworth filter performs the denoising function (2), which gives the noiseless output image. The background and blood vessels are removed $(13,14)$ from the image for accurate drusen detection. Next, the bits have been converted into qubits for the quantum channel. Automated entropy-based thresholding is given to the converted quantum state image. This automated thresholding is based on the comparison of low and high range of entropy values. The biggest challenge is to differentiate the drusen from the exudates. Some exudates also extracted with drusen, which will be reduced using the changes of thresholding values. The boundaries of the detected drusen areas are found using Canny edge detection (16). Finally, the drusen will be detected and the identified areas are marked on the input retinal fundus image. The suggested methods will be tested with the various databases (DRIVE, STARE, MESSIDOR, E-Optha-Ex and ONH-Hunter). The output image results for the 15 sample images are shown in Fig. 5. 


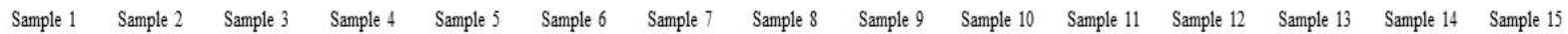

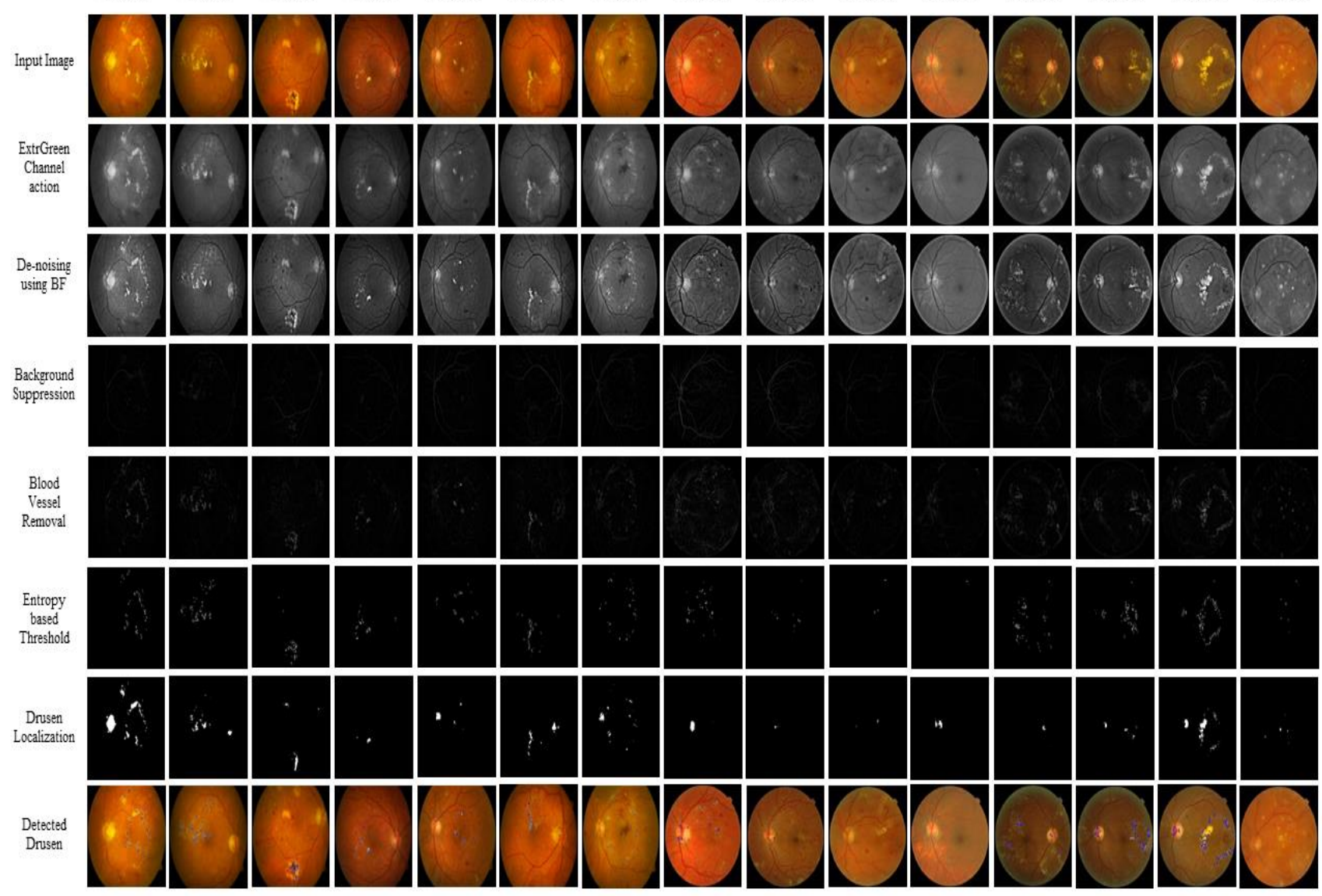

Fig. 5.The results for the databases (DRIVE, STARE, MESSIDOR, E-Optha-Ex, ONH Hunter) using a quantum-assisted retinal drusen detection algorithm

Table I: Performance analysis for the quantum-assisted drusen detection algorithm

\begin{tabular}{|c|c|c|c|c|c|}
\hline $\begin{array}{c}\text { Input } \\
\text { Fundus } \\
\text { Image }\end{array}$ & Database & Sensitivity & Specificity & Accuracy & $\begin{array}{c}\text { Execution Time } \\
\text { (Seconds) }\end{array}$ \\
\hline Image 1 & DRIVE & 0.9387 & 0.9707 & 0.9663 & 0.93 \\
\hline Image 2 & DRIVE & 0.9279 & 0.9625 & 0.9597 & 1.32 \\
\hline Image 3 & DRIVE & 0.9354 & 0.9727 & 0.9518 & 1.48 \\
\hline Image 4 & STARE & 0.9411 & 0.9862 & 0.9725 & 0.59 \\
\hline Image 5 & STARE & 0.9482 & 0.9816 & 0.9703 & 0.68 \\
\hline Image 6 & STARE & 0.9321 & 0.9758 & 0.9692 & 0.89 \\
\hline Image 7 & MESSIDOR & 0.9003 & 0.9393 & 0.9219 & 2.82 \\
\hline Image 8 & MESSIDOR & 0.9195 & 0.9569 & 0.9466 & 1.73 \\
\hline Image 9 & MESSIDOR & 0.9487 & 0.9791 & 0.9633 & 0.91 \\
\hline Image 10 & E-OPTHA Ex & 0.9372 & 0.9738 & 0.9645 & 0.89 \\
\hline Image 11 & E-OPTHA Ex & 0.9544 & 0.9917 & 0.9803 & 0.53 \\
\hline Image 12 & E-OPTHA Ex & 0.9336 & 0.9728 & 0.9619 & 0.85 \\
\hline Image 13 & ONH-Hunter & 0.9218 & 0.9623 & 0.9578 & 1.39 \\
\hline Image 14 & ONH-Hunter & 0.9171 & 0.9555 & 0.9469 & 1.63 \\
\hline Image 15 & ONH-Hunter & 0.9297 & 0.9639 & 0.9413 & 0.94 \\
\hline \multicolumn{2}{|l|}{ Average Value } & $\mathbf{0 . 9 3 2 4}$ & $\mathbf{0 . 9 6 9 7}$ & $\mathbf{0 . 9 5 8 3}$ & $\mathbf{1 . 1 7}$ \\
\hline
\end{tabular}

The values of sensitivity, specificity, accuracy and execution time are measured (17) and tabulated in Table 1. The average value of sensitivity is 0.9324 , which value is moreover higher than other existing methods, 0.8981 for [3]. For the specificity calculation, the average value is calculated as 0.9697 , which is better than [4] but not than [3] which is 0.99 .

Published By:

DOI: 10.35940/ijeat C5781.029320

Journal Website: www.ijeat.org

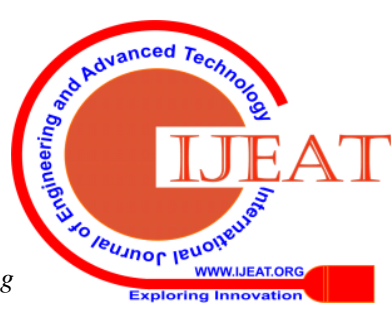


Accuracy is the main variable to decide the system will be more accurate or not. The results of our proposed method, the accuracy is 0.9583 . This value is better than all of the existing methods except [3] which are reviewed in this research work. This suggested method has been executed within 1.17 seconds averagely. From these results, the proposed quantum assisted algorithm is more efficient and lower execution time than existing drusen detection methods.

\section{CONCLUSION}

An automated drusen detection method is needed for the physicians for the diagnosis of the eye disease to reduce the time and inaccurate measurements. In this research problem, the Quantum-based retinal drusen detection method is developed under the special properties of Quantum information theory like superposition state, which strike out the limitations of the classical based algorithms. The numerical solutions and the experimental results show that the proposed algorithm performs faster than the classical systems. The values of accuracy, sensitivity and specificity are calculated and prove their efficiency over the existing algorithms. In future work, quantum-based retinal eye disease identification system will be developed.

\section{ACKNOWLEDGMENT}

This research is supported by Promotion of University Research and Scientific Excellence (PURSE) - Phase II, Department of Science and Technology (DST), Bharathiar University, Coimbatore, Tamilnadu. Award Letter No.: BU/DST PURSE (II)/ APPOINTMENT/ 9

\section{REFERENCES}

1. Paolo Checco and Fernando Corinto : CNN-Based Algorithm for Drusen Identification. IEEE, ISCAS (2006)

2. Young Jae Kim and Kwang Gi Kim : Automated Segmentation Methods of Drusen to Diagnose Age-Related Macular Degeneration Screening in Retinal Images. Hindawi Computational and Mathematical Methods in Medicine (2018)

3. Deepti Mittal, Kajal Kumari : Automated detection and segmentation of drusen in retinal fundus images. Computers and Electrical Engineering 47, 82-9 (2015)

4. Alauddin Bhuiyan, Ryo Kawasaki, Mariko Sasaki, Ecosse Lamoureux, Kotagiri Ramamohanarao, Robyn Guymer, Tien Y Wong and Kanagasingam Yogesan : Drusen Detection and Quantification for Early Identification of Age Related Macular Degeneration using Color Fundus Imaging, Bhuiyan et al., J Clin Exp Ophthalmol, 4-5 (2013)

5. Qiang Chen, Theodore Leng, Luoluo Zheng, Lauren Kutzscher, Jeffrey Ma, Luis de Sisternes , Daniel L. Rubin : Automated drusen segmentation and quantification in SD-OCT images, Medical Image Analysis 17, 1058-107 (2013)

6. A. Thaibaoui , A. Raji, P. Bunel : A Fuzzy Logic Approach to Drusen Detection in Retinal Angiographic Images, IEEE (2000)

7. Suzhen Yuan, Xia Mao, Lijiang Chen, Yuli Xue : Quantum digital image processing algorithms based on quantum measurement, Optik, 124 (2013)

8. Simona Caraiman - Vasile I. Manta : Image segmentation on a quantum computer, Quantum Inf Process (2015)

9. Zhixiao Wang, Xuebin Xu, Wenyao Yan, Wei Wei, Junhuai Li and Deyun Zhang : Optimal Scheme of Retinal Image Enhancement using Curvelet Transform and Quantum Genetic Algorithm, Ksii Transactions On Internet And Information Systems Vol. 7, No. 11, Nov. (2013)

10. Çalar Ayteki, Serkan Kiranya and Moncef Gabbouj : Quantum Mechanics In Computer Vision: Automatic Object Extraction

11. Tian Lan, Yangguang Sun, Mingyue Ding : A fast quantum mechanics based contour extraction algorithm, Proc. of SPIE Vol. 7259, Medical Imaging: Image Processing (2009)

12. Hichem Talbi, Mohamed Batouche, Amer Draa :A Quantum-Inspired Evolutionary Algorithm for Multiobjective Image Segmentation ,
International Scholarly and Scientific Research \& Innovation 1(7) (2007)

\section{AUTHORS PROFILE}

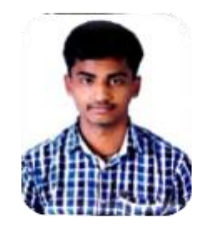

L. Jeganson Durai, received the M.Sc. and M.Phil degrees in Electronics and Instrumentation from Bharathiar University in 2015 and 2017, respectively. Currently, he is a Ph.D. research scholar in the same place. His field of research is Digital Image Processing, Quantum Computing. He has published a number of papers in international journals and presentations in

conferences.

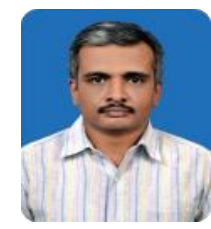

Dr. J.Vijayakumar, obtained his Bachelor of Engineering in Electronics and Communication Engineering from Maharaja Engineering College Avinashi, affiliated to Bharathiar University, Coimbatore in the year 2003. He obtained his Master of Engineering in Applied Electronics from Bannari Amman Institute of Technology - Sathyamangalam under Anna University, Chennai in the year 2005. He received Doctor of Philosophy in Information and Communication Engineering under Anna University, Chennai in the year 2015. Dr. J.Vijayakumar started his career as Lecturer at Mahendra Engineering College - Tiruchengode in the Department of Electronics and Communication Engineering from the year academic June 2005 to May 2010. He joined as Associate Professor in the Department of Electronics and Communication Engineering at Nandha College of Technology - Erode from the academic year June 2010 to November 2016. He joined as Associate Professor in the Department of Electronics and Instrumentation from Bharathiar University, Coimbatore from the academic year November 2016 to till date. He is a member in IE, IETE, ISECE \& UASEE. He has so far published over 50 papers in various National and International Journals and Conferences. His area of interest is Digital signal \& Image Processing. 